\title{
Speech evaluation in children with temporomandibular disorders
}

\author{
Raquel Aparecida PIZOLATO'1, Frederico Silva de Freitas FERNANDES², Maria Beatriz Duarte GAVIÃO
} 1- Speech-Language Pathologist, MSc, Graduate student, Department of Community Dentistry, Piracicaba Dental School, University of Campinas, Piracicaba,
SP, Brazil.
2- DDS, MSc, Graduate student, Department of Prosthodontics and Periodontology, Piracicaba Dental School, University of Campinas, Piracicaba, SP, Brazil.
3- DDS, MSc, PhD, Full Professor, Department of Pediatric Dentistry, Pediatric Dentistry Division, Piracicaba Dental School, University of Campinas, Piracicaba,
SP, Brazil.

Corresponding address: Profa. Dra. Maria Beatriz Duarte Gavião - Faculdade de Odontologia de Piracicaba - UNICAMP - Departamento de Odontologia Infantil - Área de Odontopediatria - Avenida Limeira 901 - 13414-903 - Piracicaba, SP - Brasil - Phone: $551921065200 / 21065368$ - FAX - 551921065218 e-mail: mbgaviao@fop.unicamp.br

Received: September 02, 2009 - Modification: April 30, 2010 - Accepted: October 26, 2010

\section{ABSTRACT}

\begin{abstract}
$\mathrm{O}$ bjectives: The aims of this study were to evaluate the influence of temporomandibular disorders (TMD) on speech in children, and to verify the influence of occlusal characteristics. Material and methods: Speech and dental occlusal characteristics were assessed in 152 Brazilian children ( 78 boys and 74 girls), aged 8 to 12 (mean age 10.05 \pm 1.39 years) with or without TMD signs and symptoms. The clinical signs were evaluated using the Research Diagnostic Criteria for TMD (RDC/TMD) (axis I) and the symptoms were evaluated using a questionnaire. The following groups were formed: Group TMD $(n=40)$, TMD signs and symptoms (Group $S$ and $S, n=68$ ), TMD signs or symptoms (Group $\mathrm{S}$ or $\mathrm{S}, \mathrm{n}=33$ ), and without signs and symptoms (Group N, $\mathrm{n}=11$ ). Articulatory speech disorders were diagnosed during spontaneous speech and repetition of the words using the "Phonological Assessment of Child Speech" for the Portuguese language. It was also applied a list of 40 phonological balanced words, read by the speech pathologist and repeated by the children. Data were analyzed by descriptive statistics, Fisher's exact or Chi-square tests $(\alpha=0.05)$. Results: A slight prevalence of articulatory disturbances, such as substitutions, omissions and distortions of the sibilants /s/ and /z/, and no deviations in jaw lateral movements were observed. Reduction of vertical amplitude was found in 10 children, the prevalence being greater in TMD signs and symptoms children than in the normal children. The tongue protrusion in phonemes $/ \mathrm{t} /, / \mathrm{d} /, / \mathrm{n} /, / \mathrm{l} /$ and frontal lips in phonemes /s/ and /z/ were the most prevalent visual alterations. There was a high percentage of dental occlusal alterations. Conclusions: There was no association between TMD and speech disorders. Occlusal alterations may be factors of influence, allowing distortions and frontal lisp in phonemes $/ \mathrm{s} /$ and $/ \mathrm{z} /$ and inadequate tongue position in phonemes /t/; /d/; /n/; /l/.
\end{abstract}

Key words: Speech. Temporomandibular joint disorders. Phoneme. Phonetics. Dental occlusion. Children.

\section{INTRODUCTION}

Speech is a dynamic and complex process comprising respiration, phonation, resonation, articulation, and neurologic integration. Air emitted from the lungs passes along the trachea and through the vocal tract to produce sounds. These sounds are then formulated into meaningful speech by articulation of the lips, teeth, tongue, palate, and alveolus ${ }^{10}$. Mandible participation, more specifically the mandibular movements, enable space modifications, allowing free tongue and soft tissues movements ${ }^{2,21}$. The freedom involved in these movements depends on the health and integrity of the stomatognathic system structures, mainly the temporomandibular joint (TMJ) and skeletal muscles ${ }^{2,12,13,16,18}$. The presence of temporomandibular disorders (TMD) can cause 
reduction in mandibular opening and retrusion ranges, as well as in prevalence of unilateral deviation movements during speech ${ }^{3}$, but pain severity do not seem to determine larger reduction of these values ${ }^{4}$. Conversely, phonemic distortions seem to be related also to tongue thrust ${ }^{10}$, but TMD seem to produce changes in speech articulation as a protective adaptive mechanism, probably triggered by pain 22 .

In addition, it has been suggested that speech alterations can be due to dental occlusal, muscular and intra-articular alterations in individuals with $\mathrm{TMD}^{20}$. Excessive overjet predisposes to large mandibular movements, most probably due to functional reasons, speech articulation, and bite, which may stress the masticatory muscles ${ }^{17}$.

It is well accepted that the etiology of TMD is multifactorial, but there is still a need to describe the relationship between TMD signs and symptoms and orofacial functions (mastication, swallowing, and speech) during growth, since these disorders tend to occur in the same children ${ }^{15}$ and are closely related to each other. Thus, considering that differences in study designs have impacted greatly on the findings of these relationships, added to the fluctuation in speech misarticulation with age ${ }^{17}$ and signs and symptoms of $\mathrm{TMD}^{5}$, as well as lack of any clear evidence directly relating malocclusions to speech discrepancies, further studies in this field are required. Therefore, the aim of this study was to evaluate the influence of TMD on speech in children, and to verify the influence of occlusal characteristics.

\section{MATERIAL AND METHODS}

A cross-sectional study design was used with subjects attending two public schools in Piracicaba, State of São Paulo, Brazil. Permission to carry out the research was obtained and the children's parents signed a written informed consent ${ }^{6}$. The Ethics Committee of the Piracicaba Dental School, University of Campinas, São Paulo, Brazil, approved the research (Process 034/2006). Initially, 350 children aged 8 to 12 years having the same socioeconomic background were selected. The inclusion criteria were a healthy status or absence of systemic disturbance in the masticatory system, presence of all primary and/or permanent teeth without anomalies and alterations of form, structure, or number. The exclusion criteria included anterior open bite, anterior cross bite, short lingual frenulum (when the tip of the tongue could not be protruded beyond the lower incisor teeth and the mobility of the tongue was restricted), presence of nutritive and non-nutritive sucking habits, neurological disturbances (such as cerebral palsy, movement disorders, speech and language disorders, and behavioral/cognitive syndromes), any type of orthodontic treatment prior to or during the research examination period and uncooperative behavior. Open bite was considered an exclusion criterion, since this malocclusion can determine speech alterations by itself, and so the influence of TMD could be masked, causing a bias in the results.

The parents/guardians were asked personally at the school or by phone about the presence of parafunctional habits in their children, such as bruxism, pacifier and thumb/finger sucking. After applying the exclusion criteria, 152 children (78 boys and 74 girls, mean age $10.05 \pm 1.39$ years) were enrolled in the study.

\section{Procedures}

The Research Diagnostic Criteria for TMD (RDC/TMD) exam and the speech evaluation were performed by a speech-language pathologist (author R.A.P.), who was trained by a member of the International RDC/TMD Consortium. Dental examination was performed by a dentist (author F.S.F.F).

\section{Evaluation of subjective symptoms}

A self-report questionnaire was used to assess subjective symptoms, regarding pain in the jaws when in function, unusually frequent headaches (more than once a week), stiffness/tiredness in the jaws, difficulty in opening the mouth wide, grinding teeth and TMJ sounds ${ }^{19}$. Each question required a "yes" or "no" answer. Moreover, three questions of the axis II of the Research Diagnostic Criteria for TMD (RDC/TMD) were considered for further TMD diagnosis:

(1) Have you had pain in the face, jaw, temple, in front of the ear or in the ear in the past month? (Obs.: Only pains of unknown etiology were considered)

(2) Have you ever had your jaw lock or catch so that it would not open all the way?

(3) Was this limitation in jaw opening severe enough to interfere with your ability to eat?

\section{Examination of clinical signs}

The clinical signs were assessed using the RDC/TMD criteria, including: pain on palpation, mandibular range of motion $(\mathrm{mm})$, associated pain (jaw opening pattern, unassisted opening, maximum assisted opening, mandibular excursive and protrusive movements), sounds from the $\mathrm{TMJ}$, and tenderness induced by muscle and joint palpation. The RDC/TMD criteria classify the most common forms of TMD into 3 diagnostic categories and allow for multiple diagnoses to be given for a single patient, as follows: Group I - muscle disorders (Group Ia with myofascial pain and Group Ib - myofascial pain with limited opening); 
Group II, with disc displacement (Group IIa - with reduction; Group IIb- without reduction with limited opening; Group IIc without reduction limited opening); and Group III, with arthralgia (Group IIIa) or arthritis (Group IIIb /IIIc). The RDC/TMD specifies distinct operational criteria for each TMD subtype; for example, a myalgia diagnosis is made if a person reports pain in the face or mastication muscles at rest or during function, as well as the presence of pain upon palpation of 3 or more sites. The arthralgia diagnosis includes pain upon palpation of the TMJ and joint-related pain during the movements of opening mouth, mandibular excursive and protrusive movements; the diagnosis of arthritis includes pain in addition to the reported clicking sounds upon palpation. Thus, every TMD subject could have both a masticatory muscle pain diagnosis and/or a TMJ pain diagnosis. Children presenting at least one sign and/or symptom of TMD, but not classified as having TMD in accordance with RDC/TMD, were allocated in groups "sign and symptom" or "sign or symptom", being the first one considered more severe.

\section{Evaluation of morphological occlusion}

The intraoral examination consisted of evaluation of the following occlusal characteristics in the clenching position:

Sagittal permanent first molar relationship: in accordance with Angle's classification with reference to the position of these teeth and how their biting surfaces fit together:

Class I: normal biting relationship between the maxillary and mandibular teeth and jaw (balanced bite).

Class II: The mandibular first molar is posterior or more towards the back of the mouth than the maxillary first molar.

Class III: The mandibular first molar is anterior or more towards the front of the mouth than the maxillary first molar.

Posterior crossbite: one or more teeth in the maxillary buccal segment are lingual to one or more of the opposing teeth in the mandibular buccal segment in maximum intercuspation.

Overbite: vertical overlap of maxillary over mandibular central incisor teeth $(\mathrm{mm})$. Values higher than $4 \mathrm{~mm}$ were considered morphological alteration. Children with negative values (anterior open bite) were not included (exclusion criterion).

Overjet: horizontal projection of maxillary central incisor teeth beyond the mandibular central incisor teeth $(\mathrm{mm})$. Values equal or higher than $4 \mathrm{~mm}$ were considered morphological alteration. Children with negative values (anterior cross bite) were not included (exclusion criterion).

\section{Speech evaluation}

A list of five easily recognizable pictures of the Phonological Assessment of Child Speech ${ }^{23}$, for sequential naming, containing all of the phonemes of the Brazilian Portuguese language in several positions, was used. The respective places of phoneme articulation are: bilabial: $[\mathrm{p}, \mathrm{b}, \mathrm{m}]$; labiodental: $[\mathrm{f}, \mathrm{v}]$; linguodental: $[\mathrm{t}, \mathrm{d}, \mathrm{n}]$; alveolar: $[s, z, l, r]$; palatal: $[x, j, l h, n h]$; velar: $[c(=k), q u$, $\mathrm{g}$ (=guê), rr]. The exam was undertaken with the participant seated on an armless chair, in a room with good acoustic conditions. Speech samples were obtained in a situation of spontaneous speech (visual stimulus).

After a period of interaction between speechlanguage pathologist and participant, the figures were presented one at a time to the participant together with the following request: "please, look at this figure and tell me all about it". The speech was interrupted with questions and/or comments only in cases in which it was necessary to encourage the production of speech for obtaining 125 words. A list of 40 phonological balanced words, read by the speech pathologist and repeated by the children was also applied to verify the improvement of the possible articulatory errors detected during spontaneous speech, since the repetition gives an acoustic support.

The speech samples were recorded using an audio cassette and transcribed literally, excluding the questions and comments and analyzed by a trained speech-language pathologist, who listened to the voice records for three times and made phonetic transcriptions of the words for documenting the absence or presence of the articulatory disorders.

The identification of speech disorders was based on the auditory perceptual analysis by the researcher and on the visual inspection of the tongue movements during evaluation. It was considered as results the common findings or the predominant ones among the three analyses. If a phoneme, contained in a word list, was wrongly pronounced in a rate of $50 \%$ or more, the articulatory error was computed. In those evaluations, the phonetic component was observed. The articulatory disorders were considered substitutions (when one sound is replaced by another), omissions (sound is omitted from a word), and distortions (sound is not produced appropriately, but still understood) of the phonemes. Moreover, tongue protrusion in phonemes /t/, /d/, /n/, /l/ (tongue tip positioned through the incisal edges of maxillary and mandibular anterior teeth), frontal lisp in phonemes /s/ and /z/, as well as the lateral deviations in jaw movements and reduction of vertical amplitude were observed. Lateral deviations were considered as a deviation in mandibular movement from central 
to lateral region (right or left) during speech. The vertical amplitude was considered restricted when there was articulation with small opening and decreasing of facial muscle movements, leading to an unclear speech.

\section{Statistical analysis}

Replicate measurements of clinical signs of TMD and occlusal variables were performed in twenty children randomly selected, for assessing intraexaminer reliability. For this, intraclass correlation coefficients (ICCs) were calculated for both continuous and dichotomous variables of RDC/TMD examination ${ }^{11}$. The considered ICC values were: ICC $<0.04$, poor reliability; $0.04 \leq$ ICC $\leq 0.075$, fair to good reliability; ICC $>0.75$ excellent reliability.

For occlusal variables, which were considered as dichotomous (presence or absence of alteration), the agreement between the two examinations was calculated. The interval among examinations was at least 1 week.

The groups were distributed in accordance with RDC/TMD diagnostic criteria and presence of signs and/or symptoms of TMD. Furthermore, due to the number of subjects in some groups, four major groups were formed to allow appropriate statistical analysis. Fisher's exact or Chi-square tests at $5 \%$ significance level were applied to verify the proportions of the independent variables (speech disorders and occlusal alterations) among the groups. Biostatic 4.0 package was used for data analysis.

\section{RESULTS}

Sample distribution according to TMD diagnosis and presence or absence of signs and symptoms of TMD is shown in Table 1. Most children presented at least one sign and one symptom of TMD. Only 5 children presented more than one RDC/TMD diagnosis.

Table 2 shows the distribution of children with speech disorders in each group, as well as their distribution in relation to occlusal characteristics. Although no differences were observed among groups, it was verified that Group N presented only 2 children (18\%) with two kind of speech disorders, whereas for other three groups the percentage ranged from $33 \%$ to $45 \%$. The proportion of children with overjet was significantly higher for Group TMD and Group $\mathrm{S}$ and $\mathrm{S}$ than the other two groups $(p<0.05)$. Moreover, between Group TMD and Group $S$ and $S$, the respective proportion was similar $(p>0.05)$, as well as for Group S or S and Group N $(p>0.05)$. For other occlusal characteristics, there were no differences in proportions among groups.

Table 3 shows the most prevalent speech disorders and their distribution in accordance with morphologic characteristics of occlusion. The proportions of overall speech disorders, as well as, for each one evaluated, were similar among the different occlusal characteristics. On the other hand, when excessive overjet and overbite $(\geq 4 \mathrm{~mm}$ ) and posterior crossbite were analyzed considering both molar classes together (Table 4), children with excessive overjet presented higher proportion of speech disorders than children with posterior crossbite $(p=0.0184)$, whereas for

Table 1- Sample distribution according to Research Diagnostic Criteria for Temporomandibular Disorders (RDC/TMD) diagnosis and signs and symptoms of TMD

\begin{tabular}{lccccc}
\hline & $\mathbf{n}$ & $\%$ & Boys (n) & Girls (n) & Mean age \pm SD \\
\hline (1) Group TMD & 40 & 26.32 & 20 & 20 & $9.73 \pm 1.38$ \\
Group la (myofascial pain) & 13 & 8.52 & 5 & 8 & $9.85 \pm 1.46$ \\
\hline Group Ib (myofascial pain with limited opening) & 3 & 1.97 & 1 & 2 & $9.67 \pm 1.15$ \\
Group IIla (arthralgia) & 19 & 12.5 & 12 & 7 & $9.47 \pm 1.50$ \\
Group la and IIla & 3 & 1.97 & 2 & 1 & $11 \pm 0$ \\
Group Ib and IIla & 1 & 0.65 & 0 & 1 & $9 \pm 0$ \\
Group la, Ib and IIla & 1 & 0.65 & 0 & 1 & $10 \pm 0$ \\
(2) Group Signs and symptoms of TMD (Group S and S) & 68 & 44.73 & 36 & 32 & $10.19 \pm 1.31$ \\
(3) Group Symptoms or Signs of TMD (Group S or S) & 33 & 21.7 & 18 & 15 & $10.12 \pm 1.49$ \\
Symptoms of TMD & 6 & 3.94 & 5 & 1 & $10.67 \pm 1.51$ \\
\hline Signs of TMD & 27 & 17.76 & 13 & 14 & $10.04 \pm 1.52$ \\
(4) Group Normal - without signs and symptoms of TMD (Group N) & 11 & 7.23 & 4 & 7 & $10.09 \pm 1.58$ \\
\hline Total Sample & 152 & 100 & 78 & 74 & $10.05 \pm 1.39$ \\
\hline
\end{tabular}

(1), (2), (3), (4): Major groups. SD= standard deviation 
excessive overbite the respective difference was not detected $(p=0.0633)$. Children with overjet $\geq 4 \mathrm{~mm}$ presented the same proportion of overall speech disorders than children with overbite $\geq 4$ $\mathrm{mm}(\mathrm{p}=0.6089)$. On verifying the presence and absence of malocclusions, children with overjet had a higher percentage of speech disorders than the ones without overjet $(p=0.0117)$. For overbite and crossbite this difference was not observed.

Reliability value for the RDC/TMD clinical exam ranged from fair to excellent (from 0.042 to 1.0 ). The lowest values were found for some palpation tests. The intra-examiner agreement ranged from $95 \%$ to $100 \%$ between the two dental examinations.

Table 2- Distribution of subjects with speech disorders and morphological characteristics of occlusion into groups

\begin{tabular}{lccccc}
\hline & (1) Group TMD & (2) Group S and S & (3) Group S or S & (4) Group N & Total \\
& $\mathbf{n = 4 0}$ & $\mathbf{n = 6 8}$ & $\mathbf{n = 3 3}$ & $\mathbf{n = 1 1}$ & $\mathbf{n = 1 5 2}$ \\
\hline Speech disorders & $13(33 \%)$ & $27(40 \%)$ & $15(45 \%)$ & $2(18 \%)$ & $57(38 \%)$ \\
\hline Distortions /s/ and /z/ & $6(15 \%)$ & $17(25 \%)$ & $5(15 \%)$ & $1(9 \%)$ & $29(19 \%)$ \\
\hline Substitutions and omissions & $3(8 \%)$ & $2(3 \%)$ & $1(3 \%)$ & 0 & $6(4 \%)$ \\
\hline Frontal lisp /s/ and /z/ & $7(18 \%)$ & $7(10 \%)$ & $3(9 \%)$ & $1(9 \%)$ & $18(12 \%)$ \\
Tongue thrust /t/; /d/; /n/;// & $8(20 \%)$ & $10(15 \%)$ & $5(15 \%)$ & $1(9 \%)$ & $24(16 \%)$ \\
\hline Deviations of the jaw to left or right & 0 & 0 & 0 & 0 & 0 \\
Reduction of vertical amplitude & $2(5 \%)$ & $3(4 \%)$ & $5(15 \%)$ & 0 & $10(7 \%)$
\end{tabular}

Occlusal characteristics

\begin{tabular}{lccccc} 
Class I & $27(68 \%)$ & $36(53 \%)$ & $25(76 \%)$ & $8(73 \%)$ & $96(63 \%)$ \\
Class II & $13(33 \%)$ & $31(46 \%)$ & $7(21 \%)$ & $3(27 \%)$ & $54(36 \%)$ \\
\hline Class III & 0 & $1(1 \%)$ & $1(3 \%)$ & 0 & $2(1 \%)$ \\
\hline Posterior crossbite & $7(18 \%)$ & $7(10 \%)$ & $3(9 \%)$ & $2(18 \%)$ & $19(13 \%)$ \\
\hline Overbite $\geq 2 / 3$ & $21(53 \%)$ & $43(63 \%)$ & $17(52 \%)$ & $5(45 \%)$ & $86(57 \%)$ \\
Overjet $\geq 4 \mathrm{~mm}$ & $18^{\mathrm{a}}(45 \%)$ & $43^{\mathrm{a}}(63 \%)$ & $11^{\mathrm{b}}(33 \%)$ & $1^{\mathrm{b}}(9 \%)$ & $73(48 \%)$ \\
\hline
\end{tabular}

Values followed by different superscript small letters differ significantly ( $p<0.05$ Fisher's Exact test or Qui-square)

Table 3- Distribution of children with the most prevalent speech disorders according to morphological characteristics of occlusion

\begin{tabular}{|c|c|c|c|c|c|c|c|c|c|c|}
\hline & & \multicolumn{4}{|c|}{ Class I } & \multicolumn{4}{|c|}{ Class II } & \multirow[t]{2}{*}{ Crossbite } \\
\hline & & \multicolumn{2}{|c|}{ Overjet ( $\geq 4 \mathrm{~mm}$ ) } & \multicolumn{2}{|c|}{ Overbite ( $\geq 4 \mathrm{~mm}$ ) } & \multicolumn{2}{|c|}{ Overjet ( $\geq 4 \mathrm{~mm}$ ) } & \multicolumn{2}{|c|}{ Overbite ( $\geq 4 \mathrm{~mm}$ ) } & \\
\hline & & Absent & Present & Absent & Present & Absent & Present & Absent & Present & \\
\hline & Total & $n=65$ & $n=32$ & $(n=46)$ & $(n=50)$ & $n=2$ & $n=41$ & $(n=18)$ & $(n=36)$ & $n=19$ \\
\hline $\begin{array}{l}\text { Speech } \\
\text { disorders }\end{array}$ & 57 & $\begin{array}{c}17 \\
(30 \%)\end{array}$ & $\begin{array}{c}14 \\
(25 \%)\end{array}$ & $\begin{array}{c}14 \\
(25 \%)\end{array}$ & $\begin{array}{c}17 \\
(30 \%)\end{array}$ & $2(4 \%)$ & $23(40 \%)$ & $9(16 \%)$ & $17(30 \%)$ & $6(11 \%)$ \\
\hline $\begin{array}{l}\text { Distortions /s/ } \\
\text { and /z/ }\end{array}$ & 29 & $6(21 \%)$ & $9(31 \%)$ & $5(17 \%)$ & $9(31 \%)$ & $2(7 \%)$ & $12(41 \%)$ & $3(10 \%)$ & $12(41 \%)$ & $3(10 \%)$ \\
\hline $\begin{array}{l}\text { Frontal lisp /s/ } \\
\text { and }|z|\end{array}$ & 18 & $4(22 \%)$ & $4(22 \%)$ & $4(22 \%)$ & $4(22 \%)$ & $2(11 \%)$ & $8(44 \%)$ & $7(39 \%)$ & $3(17 \%)$ & $2(11 \%)$ \\
\hline $\begin{array}{l}\text { Tongue thrust /t/; } \\
\qquad / \mathrm{d} / ; / \mathrm{n} / ; / \mathrm{l} /\end{array}$ & 24 & $5(21 \%)$ & $5(21 \%)$ & $7(29 \%)$ & $3(13 \%)$ & $2(8 \%)$ & $12(50 \%)$ & $8(33 \%)$ & $6(25 \%)$ & $4(17 \%)$ \\
\hline $\begin{array}{l}\text { Reduction } \\
\text { of vertical } \\
\text { amplitude }\end{array}$ & 10 & $6(60 \%)$ & $1(10 \%)$ & $4(40 \%)$ & $3(30 \%)$ & 0 & $3(30 \%)$ & 0 & $3(30 \%)$ & 0 \\
\hline $\begin{array}{l}\text { Substitutions } \\
\text { and omissions }\end{array}$ & 6 & $1(17 \%)$ & $1(17 \%)$ & $1(17 \%)$ & $2(33 \%)$ & $2(33 \%)$ & $2(33 \%)$ & $1(17 \%)$ & $2(33 \%)$ & $1(17 \%)$ \\
\hline
\end{tabular}


Table 4- Distribution of children with the overall speech disorders in accordance with morphological characteristics of occlusion independent of molar relationship

\begin{tabular}{|c|c|c|c|c|c|c|c|}
\hline & \multicolumn{2}{|c|}{ Overjet ( $\geq 4 \mathrm{~mm}$ ) } & \multicolumn{2}{|c|}{ Overbite ( $\geq 4 \mathrm{~mm}$ ) } & \multicolumn{3}{|c|}{ Posterior crossbite } \\
\hline & Absent & Present & Absent & Present & Absent & Present & \\
\hline \multicolumn{8}{|l|}{ Speech disorders } \\
\hline & $19(34 \%)^{*}$ & $37(66 \%)^{*} \dagger$ & $23(40 \%)$ & $34(60 \%)$ & $13(89 \%)$ & $6(11 \%) \dagger$ & $t p=0.0184$ \\
\hline & \multicolumn{2}{|c|}{${ }^{*} p=0.0117$} & \multicolumn{2}{|c|}{$p=0.8856$} & \multicolumn{2}{|c|}{$p=0.0608$} & \\
\hline
\end{tabular}

†Overjet $\geq 4 \mathrm{~mm}$ determined higher proportion of speech disorders than posterior crossbite $(p=0.0184)$

${ }^{*}$ Overjet $\geq 4 \mathrm{~mm}$ determined higher proportion of speech disorders than normal overjet $(p=0.0117)$

\section{DISCUSSION}

The prevalence of children diagnosed as having TMD by RDC/TMD criteria was $26.32 \%$ (Table 1 ). Moreover, most of the children presented at least one sign and/or symptom of TMD, without gender differences, in agreement with Farsi ${ }^{8}$ (2003). This finding might merit some thought since different results in the literature about TMD in children can be found. The know fluctuation in signs and symptoms of musculoskeletal disorders in a timedependent context might be a factor of influence ${ }^{5}$, as well as the dynamism of the stomatognathic system development ${ }^{12}$. Signs and symptoms in growing individuals may be due in part to growth changes ${ }^{17}$, phase in which there are both local and central factors associated from time to time with TMD development. However, the prediction of single TMD signs for the development of severe disorder later in life is unclear. Moreover, most of signs and symptoms are mild5,14 and it is not yet clear whether these represent normal variation, preclinical features or manifestations of a disease state ${ }^{1}$. Although TMD signs and symptoms fluctuated, the intraexaminer reliability showed confident results for the collected data. The palpation tests, as found also by Lobbezzoo, et al.11 (2005), showed the lowest ICCS, which can have generate some overestimation of TMD signs and symptoms, but not invalidating the classification adopted, since the signs and symptoms can fluctuate ${ }^{5}$.

A low prevalence of speech disorders was observed in all defined groups (Table 2), but it was greater in children with TMD signs and symptoms compared to the TMD-free ones. The speech evaluation was carried out by a single examiner (R.A.P.) could be considered as a limitation in the present study, but the fact that the voices were listened 3 times, gives a support for reliable results. On the other hand, this fact could be considered as a limitation, since more additional judges would provide a measure of inter-examiner agreement, eliminating possible bias. The results of the present study differ from those of Pahkala, et al. ${ }^{15}$ (1991), who found a higher frequency of subjective symptoms and several clinical signs of TMD related to certain articulatory speech disorders. The controversial results are probably due to the differences in subjects' ages in the two studies and because their children were referred for speech therapy, whereas in the present study the sample was randomly selected, without considering the severity of speech disorders. Moreover, they considered that expression of TMD and disorders in speech sound production seem to reflect, to a considerable extent, immaturity of fine motor control of the orofacial muscles in 6-8-year-old children, but in the age range of the sample in the present study, it was expected that all sounds would be produced correctly.

In the TMD and TMD signs and/or symptoms groups, a slight prevalence of articulatory disorders, such as substitutions, omissions, and distortions of the sibilants /s/ and /z/ was observed. Moreover, there were no different proportions among the four groups for these variables ( $p>0.05)$, meaning that in the studied sample, the alterations could not be associated with TMD, but with other factors.

In the visual assessment, the most prevalent alterations were tongue thrust in $/ \mathrm{t} /, \mathrm{d} / \mathrm{,} / \mathrm{n} /$, $/ \mathrm{I} /$ and frontal lisp in /s/ and /z/, but there was no difference in proportion among groups. The Class II relationship with excessive overjet may be one of the main factors responsible for frontal lisping ${ }^{7}$. In this study, the number of children with excessive overjet and overall speech disorders was significantly higher than children with normal overjet, but no differences with other malocclusion were noted (Tables 3 and 4). This can be attributed to the low prevalence of speech disorders, since the sample was randomly selected, as considered above. Moreover, distortions in /s/ and / $\mathrm{z} /$ were more prevalent in children with overjet or overbite in both class molar relationship, showing that malocclusion can play a role in some speech alterations, aside others factors of influence (Table 3).

No deviations in jaw lateral movements were observed during the sequential naming of pictures in the present sample, whereas reduction of vertical amplitude was found in the TMD and $S$ and $S$ groups, but only in 10 children. Thus, the reference 
of functional limitations due to the characteristics of TMD in the studied sample was not confirmed, in agreement with others ${ }^{4,9}$. Nonetheless, a discrete number of alterations were assessed visually, which could mean a modulation of motor responses preserving the function ${ }^{9}$ in children with TMD and signs and/or symptoms of TMD in this study.

Although a large number of children were diagnosed with TMD/RDC and signs and/or symptoms of TMD, a small number presented articulatory errors. This suggests either that the errors were undetectable by perceptual and acoustic evaluation or that these subjects had developed some kind of compensatory pattern ${ }^{1}$. In this way, it is important to emphasize, as stated above, the random sample characteristic, since the children were selected from public schools, not taking into consideration the severity of speech disorders. In addition, the quite strict exclusion criteria of the sample can be considered as influence factors on the results. Bianchini, et al. ${ }^{3}$ (2008) established no statistically significant differences for the presence and the range of the deviations in laterality, assessed by computerized electrognathography, during speech between TMD and asymptomatic adult subjects. However, these authors considered that the presence of TMD could determine reduction in mandibular opening and prevalence of unilateral deviation movements during speech. In spite of the differences in results, it should be emphasized that attention should be given to children with TMD and signs and symptoms of $\mathrm{TMD}^{5}$, in order to identify patients who should be more closely observed, in view of the high prevalence found in the present study.

\section{CONCLUSION}

There was no association between TMD and speech disorders. Occlusal alterations may be factors of influence, allowing distortions and frontal lisp in phonemes $/ \mathrm{s} /$ and $/ \mathrm{z} /$ and inadequate tongue position in phonemes $/ \mathrm{t} / ; / \mathrm{d} / ; / \mathrm{n} / ; / \mathrm{l}$.

\section{REFERENCES}

1- AAPD American Academy of Pediatric Dentistry. 2010-2011 definitions, oral health policies, and clinical guidelines [cited 25 Jan. 2011]. Available from: <http://www.aapd.org/media/ Policies_Guidelines/G_TMD.pdf>

2- Bianchini EM, Andrade CR. A model of mandibular movements during speech: normative pilot study for the Brazilian Portuguese language. Cranio. 2006;24:197-206.

3- Bianchini EM, Paiva G, Andrade CR. Mandibular movement patterns during speech in subjects with temporomandibular disorders and in asymptomatic individuals. Cranio. 2008;26:50-8. 4- Bianchini EM, Paiva G, Andrade CR. Mandibular movements in speech: interference of temporomandibular dysfunction according to pain indexes. Pro Fono. 2007;19:7-18.
5- Bonjardim LR, Gavião MB, Pereira LJ, Castelo PM, Garcia RC. Signs and symptoms of temporomandibular disorders in adolescents. Braz Oral Res. 2005;19:93-8.

6- Brazil. Health Ministry. National Health Council. Resolution 196, 10 Oct. 1996. On research involving human subjects. Federal Official Gazette of Brazil.

7- Farret MM, Jurach EM, Brandão L, Moraes DC, Brandão SR, Santos SL. Relationship between malocclusion and fonoarticulatory disorders. Int J Orofacial Myology. 1998;24:20-6.

8- Farsi NM. Symptoms and signs of temporomandibular disorders and oral parafunctions among Saudi children. J Oral Rehabil. 2003;301:1200-8.

9- Garcia AR, Madeira MC, Paiva G, Olivieri KA. Joint vibration analysis in patients with articular inflammation. Cranio. 2000;18:272-9.

10- Hassan T, Naini FB, Gill DS. The effects of orthognathic surgery on speech: a review. J Oral Maxillofac Surg. 2007;65:2536-43.

11- Lobbezoo F, Van Selms MK, John MT, Huggins K, Ohrbach $\mathrm{R}$, Visscher CM, et al. Use of the Research Diagnostic Criteria for Temporomandibular Disorders for multinational research: translation efforts and reliability assessments in The Netherlands. J Orofac Pain. 2005;19:301-8.

12- Luz JGC, Maragno IC, Martin MC. Characteristics of chief complaints of patients with temporomandibular disorders in a Brazilian population. J Oral Rehabil. 1997;24:240-3.

13- Mongini F. Influence of function on temporomandibular joint remodeling and degenerative disease. Dent Clin North Am. 1983;27:479-94.

14- Nilner M, Lassing SA. Prevalence of functional disturbances and diseases of the stomatognathic system in 7-14 year olds. Swed Dent J. 1981;5:173-87.

15- Pahkala R, Laine T, Närhi M. Associations among different orofacial dysfunctions in 9-11-year-olds. Eur J Orthod. 1995; 17:497-503.

16- Pahkala R, Laine T, Närhi M, Ettala-Ylitalo UM. Relationship between craniomandibular dysfunction and pattern of speech sound production in a series of first-graders. Eur J Orthod. 1991; 13:378-85.

17- Pahkala R, Qvarnström M. Can temporomandibular dysfunction signs be predicted by early morphological or functional variables? Eur J Orthod. 2004;26:367-73.

18- Pahkala RH, Laine-Alava MT. Changes in TMD signs and in mandibular movements from 10 to 15 years of age in relation to articulatory speech disorders. Acta Odontol Scand. 2000;58:2728.

19- Riolo ML, Brandt D, TenHave TR. Associations between occlusal characteristics and signs and symptoms of TMJ dysfunction in children and young adults. Am J Orthod Dentofacial Orthop. 1987;92:467-77.

20- Slavicek R. Relationship between occlusion and temporomandibular disorders: implications for the gnathologist. Am J Orthod Dentofacial Orthop. 2011;139:10, 12, 14.

21- Smith A, Zelaznik HN. Development of functional synergies for speech motor coordination in childhood and adolescence. Dev Psychobiol. 2004;45:22-33.

22- Suzuki E, Ishigaki S, Yatani H, Morishige E, Uchida M. Mean power frequency during speech in myalgia patients. J Oral Rehabil. 2010;37:692-7.

23- Yavas MS, ed. Phonological disorders in children: theory, research and practice. London: Routledge; 1991. 